\title{
Granulomatosis-associated common variable immunodeficiency disorder: a case-control study versus sarcoidosis
}

\author{
Diane Bouvry, Luc Mouthon, Pierre-Yves Brillet, Marianne Kambouchner, \\ Jean-Pierre Ducroix, Vincent Cottin, Julien Haroche, Jean-Francois Viallard, \\ Romain Lazor, François Lebargy, Abdellatif Tazi, Benoît Wallaert, Amar Smail, \\ Jean-Luc Pellegrin, Hilario Nunes, Zahir Amoura, Jean-François Cordier, \\ Dominique Valeyre, Jean-Marc Naccache and the Groupe Sarcoïdose Francophone
}

ABSTRACT: The aim of the present study was to investigate to what extent interstitial lung disease (ILD) in common variable immunodeficiency disorder (CVID)-associated granulomatous disease (GD) is similar to pulmonary sarcoidosis

20 patients with CVID/GD were included in a retrospective study conducted by the Groupe Sarcoïdose Francophone. Medical records were centralised. Patients were compared with 60 controls with sarcoidosis.

Clinical examination showed more frequent crackles in patients than controls (45\% versus $1.7 \%$, respectively; $\mathbf{p}<\mathbf{0 . 0 0 1}$ ). On thoracic computed tomography scans, nodules (often multiple and with smooth margins), air bronchograms and halo signs were more frequent in patients than controls ( $80 \%$ versus $42 \%$, respectively; $p=0.004$ ) as well as bronchiectasis $(65 \%$ versus $23 \%$, respectively; $p<0.001$ ). The micronodule distribution was perilymphatic in $100 \%$ of controls and in $42 \%$ of patients $(p<0.001)$. Bronchoalveolar lavage analysis showed lower T-cell CD4/CD8 ratios in patients than in controls (mean \pm SD $1.6 \pm 1.1$ versus $5.3 \pm 4$, respectively; $p<0.01$ ). On pathological analysis, nodules and consolidations corresponded to granulomatous lesions with or without lymphocytic disorders in most cases. Mortality was higher in patients than controls $(30 \%$ versus $0 \%$, respectively) and resulted from common variable immunodeficiency complications.

ILD in CVID/GD presents a specific clinical picture and evolution that are markedly different from those of sarcoidosis.

KEYWORDS: Granuloma, immunodeficiency, immunoglobulin deficiency, interstitial lung disease, sarcoidosis

ommon variable immunodeficiency disorder (CVID) is characterised by primary defective immunoglobulin production [1]. The main known clinical respiratory features in CVID are recurrent bacterial infections and bronchiectasis. CVID can be associated with autoimmune manifestations and/or granulomatous disease (GD) and/or lymphoproliferative disorders [2-4]. The association of CVID with GD (CVID/GD) was reported in $8-22 \%$ of patients in large series of CVID patients [2, 3, 5, 6]. Previous studies have suggested that GD could confer a poorer prognosis for CVID patients and that the lung is the most prominent localisation of the granulomatous process $[5,7]$.
Several questions remain concerning interstitial lung disease (ILD) in CVID/GD (ILD/CVID/GD) patients, as this condition has never been specifically and comprehensively studied on a sufficiently large scale. Most published studies on lung manifestations in CVID/GD were limited to one aspect, such as computed tomography (CT) at presentation, and/or included only a limited number of cases [7-13].

The extent to which ILD/CVID/GD is similar to pulmonary sarcoidosis and how ILD can impact the prognosis of patients with CVID and GD [7-10] is still being discussed. To date, there is no published case-control study comparing ILD/CVID/ GD and patients with pulmonary sarcoidosis.
AFFILIATIONS

For a list of the authors' affiliations, please see the Acknowledgements section.

CORRESPONDENCE

J-M. Naccache

Service de Pneumologie

Hôpital Avicenne

125 rue de Stalingrad

93009 Bobigny

France

E-mail: jean-marc.naccache@

avc.aphp.fr

Received:

Oct 302011

Accepted after revision:

April 262012

First published online:

Aug 162012 
We present a case-control study of ILD/CVID/GD versus pulmonary sarcoidosis. The objectives of the study were to describe clinical characteristics, radiological, functional, biological and pathological features, and outcomes including radiological imaging and mortality in patients with ILD/ CVID/GD, and to compare them with patients with pulmonary sarcoidosis.

\section{METHODS}

\section{Patients with ILD/CVID/GD}

The study was conducted by the Groupe Sarcoïdose Francophone, a collaborative official working group of the Société de Pneumologie de Langue Française. Physicians participating in the network were asked to report patients in their care who fulfilled the following inclusion criteria: 1) CVID diagnosed according to CONLEY et al. [1]; 2) histological evidence of noncaseating granulomas in one or more tissue samples with exclusion of any other cause of granulomatosis; and 3) presence of ILD on CT. For a complete list of the study population and hospitals involved see the online supplementary material.

The current retrospective multicentre study received institutional review board approval (Comité de Protection des Personnes d'Ile de France, Paris, France). The informed consent of patients was obtained.

The study was conducted between October 2006 and December 2010. 28 subjects were recruited. For all patients, complete medical records were collected in a centralised database from referring physicians through a detailed questionnaire from which D. Bouvry and L. Mouthon retrospectively reviewed the medical, biological and pathological records, while all chest CT scans were reviewed by P-Y. Brillet and D. Bouvry. Eight out of 28 patients were not included in this review because of the absence of confirmation of ILD on CT $(n=4)$, the absence of evidence of granulomatous lesions $(n=2)$, hypogammaglobulinaemia related to non-Hodgkin's lymphoma $(n=1)$ or the absence of informed consent $(n=1)$, so the study population included 20 patients. Disease evolution was tracked from the date of the first chest CT demonstrating ILD.

\section{Controls with pulmonary sarcoidosis}

The control group consisted of 60 patients with pulmonary sarcoidosis diagnosed according to American Thoracic Society/European Respiratory Society/World Association of Sarcoidosis and Other Granulomatous Diseases criteria [14]. Controls were matched with ILD/CVID/GD patients $(3 / 1$ ratio) for radiographic staging of sarcoidosis [14]: ILD/CVID/ GD patients with stage 1 disease were matched with stage 1 sarcoidosis patients; stages 2 and 3 were matched with stage 2 or 3 ; and stage 4 patients were matched with stage 4 sarcoidosis controls.

\section{Clinical and laboratory investigations}

For all patients, D. Bouvry and L. Mouthon retrospectively reviewed the database: clinical records including thoracic and extra-thoracic involvement, pulmonary function tests, biological results including blood and bronchoalveolar lavage (BAL), pathological records, and outcome. Available pathological specimens were reviewed by M. Kambouchner and allowed the study of CT-pathology correlations in cases of halo signs on CT.

\section{Imaging investigations}

Imaging evaluation included chest radiography and thinsection CT. Chest radiograms were analysed using the radiographic staging of sarcoidosis [14]. For thoracic CT analysis, both mediastinal and lung window settings images were reviewed in consensus by D. Bouvry and P-Y. Brillet. The observers evaluated the presence, extent and lobar distribution of the CT findings according to the Fleischner Society recommendations [15]. For further information on the imaging techniques used see supplementary material.

\section{Assessment of outcome}

Patients were regularly assessed, at time intervals depending on their clinical status. The outcome evaluation was focused on $\mathrm{CT}$, with a special emphasis on fibrosis signs and survival.

\section{Study design}

Initially, we analysed the clinical, biological, radiological, pathological and outcome data of patients with ILD/CVID/ GD. These characteristics were then compared to those of the control group with pulmonary sarcoidosis.

\section{Statistical analysis}

The results are expressed as percentage, mean \pm SD or median (range) as appropriate. Patients with ILD/CVID/GD were compared with controls by the Chi-squared test, Fisher's exact test or unpaired t-tests when appropriate. Survival of patients and control was estimated by the Kaplan-Meier method and compared with the log-rank test. p-values $<0.05$ were considered significant.

\section{RESULTS}

\section{Clinical and laboratory findings}

Patient characteristics are summarised in table 1.

The ILD/CVID/GD group consisted of 11 males and nine females. There were 18 (90\%) Caucasian and two (10\%) black patients. CVID, GD and ILD were diagnosed within 2 yrs of each for $65 \%$ of patients. The patient mean \pm SD age (range) at CVID, GD and ILD diagnosis was $42 \pm 16(11-71), 43 \pm 16$ (11-71) and $44 \pm 17$ (11-71) yrs, respectively (nonsignificant).

$13(65 \%)$ patients had a history of recurrent infections and eight $(40 \%)$ patients complained of symptoms linked to autoimmune manifestations.

GD diagnosis was confirmed in all patients by the evidence of granulomatous lesions on pathological examination in one, two or three sites for 11, seven and two patients, respectively. Pathological specimens evidencing granulomas were taken from thoracic samples in $12(60 \%)$ patients (bronchi, $n=3$; mediastinal lymph nodes, $n=4$; lung parenchyma, $n=6$ ), spleen samples in four patients, liver in four patients, salivary glands in three, skin in three, peripheral lymph nodes in two, abdominal lymph nodes in two and bone marrow in one patient. Moreover, lung parenchymal histology analysis performed in seven patients showed: 1) granulomas alone $(n=4)$; 2) granulomas associated with other lesions $(n=2)$ (organising pneumonia, $n=1$; follicular bronchiolitis, $n=1$ ); or 3) lymphoid interstitial pneumonia alone $(n=1)$. Lymphocytic phenotypes of lung infiltrates showed a predominance of Tcells mainly of the CD4 type except for the lymphocytic 


\begin{tabular}{|c|c|c|c|c|c|c|}
\hline \multirow{2}{*}{$\begin{array}{l}\text { TABLE } \\
\text { Patient }\end{array}$} & \multicolumn{6}{|c|}{$\begin{array}{l}\text { Patient characteristics at the time of diagnosis of interstitial lung disease in combined variable immunodeficiency } \\
\text { disorder associated with granulomatous disease (GD) }\end{array}$} \\
\hline & Sex/ethnicity & $\begin{array}{l}\text { Recurrent } \\
\text { infections }\end{array}$ & Autoimmune disorder & Respiratory symptoms & Auscultation & $\begin{array}{c}\text { Extrathoracic } \\
\text { involvement of GD }\end{array}$ \\
\hline 1 & $M / C$ & $\mathrm{~N}$ & $\mathrm{~N}$ & $\mathrm{D}$ & Crackles & Skin \\
\hline 2 & $M / C$ & $\mathrm{~N}$ & $\mathrm{~N}$ & $\mathrm{~N}$ & Crackles & Liver, spleen \\
\hline 5 & $M / c$ & Y & $\mathrm{N}$ & $\mathrm{N}$ & $n$ & Liver, spleen, aLN, BM \\
\hline 6 & $\mathrm{~F} / \mathrm{C}$ & $\mathrm{N}$ & Thrombocytopenia, anaemia & $\mathrm{N}$ & $n$ & Spleen, aLN \\
\hline 7 & $\mathrm{~F} / \mathrm{C}$ & Y & $\mathrm{N}$ & N & $\mathrm{n}$ & Skin, pLN \\
\hline 8 & $M / c$ & Y & $\mathrm{N}$ & $D, C$ & $n$ & Liver, spleen, eyes, PFNP \\
\hline 9 & $M / C$ & N & $\mathrm{N}$ & D & Crackles & Liver, spleen \\
\hline 13 & $M / C$ & Y & Neutropenia & $D, C$ & Crackles & Liver, spleen \\
\hline 14 & $M / C$ & Y & N & N & $n$ & $\begin{array}{c}\text { Spleen, eyes, parotitis, } \\
\text { CNS }\end{array}$ \\
\hline 15 & $M / c$ & Y & Lupus & $\mathrm{N}$ & ND & Liver, spleen, aLN \\
\hline 16 & $M / b$ & Y & $\mathrm{N}$ & N & n & Spleen, pLN \\
\hline 17 & $M / c$ & Y & Anaemia, neutropenia & $\mathrm{D}$ & Crackles & None \\
\hline 18 & $\mathrm{~F} / \mathrm{C}$ & Y & N & $D, C$ & Crackles & Spleen, skin, aLN \\
\hline 19 & $\mathrm{~F} / \mathrm{C}$ & $\mathrm{N}$ & Thrombocytopenia & D & n & Liver, spleen, eyes, CNS \\
\hline 20 & $\mathrm{~F} / \mathrm{C}$ & N & $\mathrm{N}$ & N & $n$ & Spleen \\
\hline
\end{tabular}

M: male; F: female; c: Caucasian; b: black; N: no; Y: yes; D; dyspnoea; C: cough; n: normal; ND: not determined; p: peripheral; LN: lymph node; a: abdominal; BM: bone marrow; PFNP: peripheral facial nerve paralysis; CNS: central nervous system.

interstitial pneumonia patient, who showed a predominance of CD8 T-cells. Molecular searches for clonality were negative.

Pulmonary symptoms were observed in 12 (60\%) patients and consisted of exertional dyspnoea $(n=12,60 \%)$, which was associated with productive cough in six (30\%) patients. Upon physical examination, inspiratory crackles were found in nine $(45 \%)$ patients. Splenomegaly and hepatomegaly were found in $15(75 \%)$ and $10(50 \%)$ patients, respectively.

Pulmonary function tests were normal in five (23.8\%) patients. A restrictive ventilatory defect (total lung capacity $<80 \%$ predicted) was found in seven (33.3\%) patients and an obstructive defect (forced expiratory volume in $1 \mathrm{~s} /$ vital capacity $<70 \%$ ) in two $(10 \%)$ patients. An impairment of carbon monoxide diffusing capacity $(<80 \%)$ was observed in $14(70 \%)$ patients.

Serum angiotensin-converting enzyme level was higher than the upper limit of normal (ULN) in 14 (87.5\%) out of 16 tested patients (mean $2.2 \pm 1.2 \times \mathrm{ULN}$, range $1-5 \times \mathrm{ULN}$ ). The BAL differential cell count was lymphocytic (lymphocytes $\geqslant 20 \%$ ) in $11(85 \%)$ out of 14 tested patients and mean \pm SD lymphocyte count was $37.3 \pm 15.3 \%$. The CD4/CD8 T-cell ratio, tested in 10 patients, was $<1$ in five $(50 \%)$ subjects, $1-3.5$ in four $(40 \%)$ subjects and $>3.5$ in one $(10 \%)$ subject. Mean \pm SD CD4/CD8 Tcell ratio was $1.6 \pm 1.1$ in BAL.

Extrathoracic involvement of GD, detailed in table 1, was present in $19(95 \%)$ patients with ILD/CVID/GD. The mean \pm sD number of extrathoracic localisations was $2.4 \pm 1.3$. The most frequent extrathoracic localisations were the spleen $(n=17)$, liver $(n=9)$, lymph nodes $(n=9)$, skin $(n=3)$ and eye $(n=3)$.

A comparison of the patients and controls is presented in table 2. It confirms that recurrent infections and autoimmune disease were common in CVID and rare or absent in sarcoidosis. From a clinical point of view, ILD/CVID/GD showed more frequent crackles, splenomegaly, hepatomegaly and extrathoracic localisations. Biological data showed that the BAL CD4/ CD8 ratio was lower in patients than in controls $(p<0.001)$.

\section{Radiological findings}

Using the radiographic staging for sarcoidosis, patients with ILD/CVID/GD had a chest radiographic stage of 1 for one (5\%) patient, stage 2 for eight (40\%) patients and stage 3 for 11 $(55 \%)$ patients, while there were no stage 4 patients.

The most common CT finding in ILD/CVID/GD was nodules, which were present in $16(80 \%)$ patients. In most cases, nodules were multiple $(n=10,62.5 \%)$, with air bronchograms $(n=15$, $94 \%)$ and smooth margins $(n=12,75 \%)$, and surrounded by ground-glass opacities (halo sign) ( $n=6,37.5 \%)$ (fig. 1). Micronodules were present in $12(60 \%)$ patients, had a random distribution in seven $(64 \%)$ cases and were perilymphatic in only five $(36 \%)$ cases. Septal thickening and other lines were observed in 13 (65\%) patients, without distortion (fig. 2). Bronchiectasis was seen in $13(65 \%)$ patients; it was more 


\begin{tabular}{|c|c|c|c|}
\hline & ILD/CVID/GD & Sarcoid controls & p-value \\
\hline Subjects & 20 & 60 & \\
\hline Age yrs & $44 \pm 17$ & $43 \pm 11.5$ & 0.89 \\
\hline Males/females & $11(55) / 9(45)$ & $18(30) / 42(70)$ & 0.04 \\
\hline Autoimmune disease & $8(40)$ & $1(1.7)$ & $<0.001$ \\
\hline Crackles & $9(45)$ & $1(1.7)$ & $<0.001$ \\
\hline Splenomegaly & $15(75)$ & $5(8.3)$ & $<0.001$ \\
\hline Hepatomegaly & $10(50)$ & $5(8.3)$ & $<0.001$ \\
\hline Extrathoracic localisation & $19(95)$ & $39(65)$ & 0.009 \\
\hline Restrictive syndrome & $7(35)$ & $11(18)$ & 0.1 \\
\hline$T L, C O$ impairment & $13(65)$ & $44(73)$ & 0.6 \\
\hline Blood lymphocyte count cells per $\mathrm{mm}^{3}$ & $1498 \pm 864$ & $1374 \pm 547$ & 0.57 \\
\hline Increased SACE & $14(88)$ & $47(78)$ & 0.55 \\
\hline \multicolumn{4}{|l|}{ BAL } \\
\hline BAL lymphocyte count \% & $37.3 \pm 15.3$ & $27 \pm 21$ & 0.08 \\
\hline CD4/CD8 T-cell ratio & $1.6 \pm 1.1$ & $5.3 \pm 4$ & $<0.001$ \\
\hline
\end{tabular}

Data are presented as $n$, mean $\pm S D$ or $n(\%)$, unless otherwise stated. PFT: pulmonary function test; $T L, C O$ : transfer factor of the lung for carbon monoxide; $S A C E$ : serum angiotensin-converting enzyme; BAL: bronchoalveolar lavage. Bold indicates statistically significant $p$-values.

frequently distal and involved lower lobes. Two patients had focal and cystic bronchiectasis.

The main CT pattern in ILD/CVID/GD was nodules $(n=6$, $30 \%)$, being more frequent than micronodules $(n=5,25 \%)$, lines $(n=4,20 \%)$ and ground glass, either isolated $(n=1,5 \%)$, or associated with lines $(n=3,15 \%)$ or nodules $(n=1,5 \%)$.

With regard to CT-pathological correlations, three out of six patients with a nodule surrounded by ground-glass opacity had a pathological analysis performed. In one case, reviewed by M. Kambouchner, there was a solid area of granulomas and a peripheral area showing organising pneumonia (fig. 3). The two other cases presented granulomas only. Among five
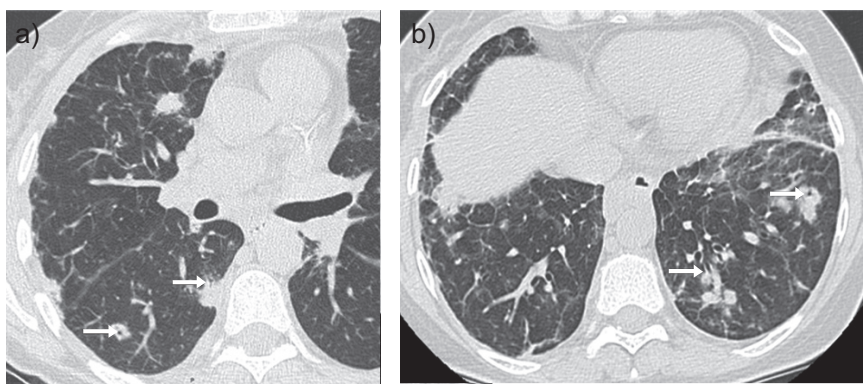

FIGURE 1. Axial transverse computed tomography images of patient 11 showing multiple nodules with air bronchograms (arrows) and smooth margins. This was associated with septal and nonseptal lines. a, b) Different lobes of the same patient showing the same pattern of lesions. patients with consolidation, three had a pathological analysis performed. All had granulomas, isolated in two cases and associated with follicular bronchiolitis in the other.

The comparison between patients and sarcoid controls is summarised in table 3.57 CT scans were analysed. Nodules

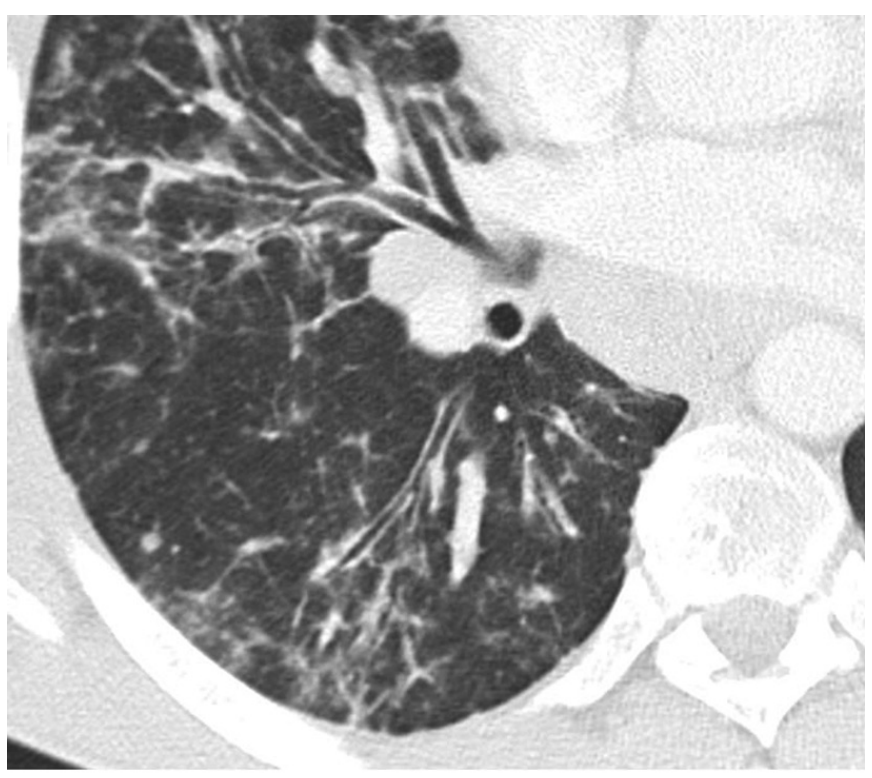

FIGURE 2. Axial transverse computed tomography images of patient 6 showing nonseptal lines with mild bronchiectasis in the middle lobe. 

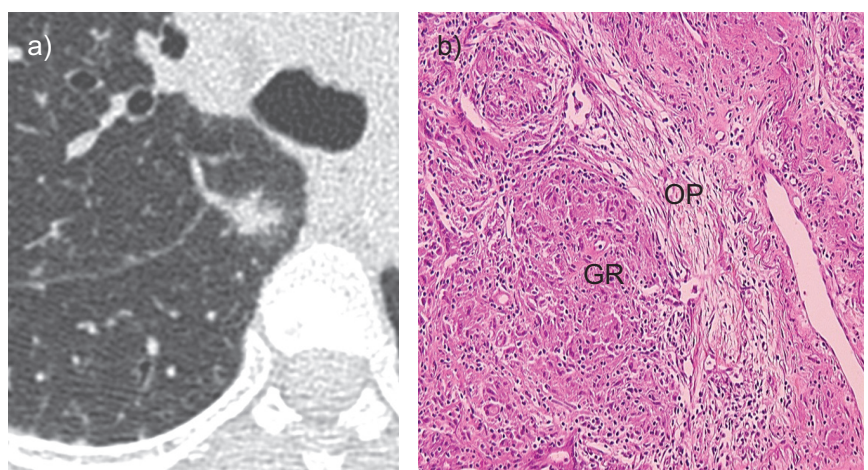

FIGURE 3. a) Axial transverse computed tomography image of patient 12 showing a nodule surrounded by a ground-glass opacity and b) corresponding pathological features. b) High magnification $(250 \times)$ of a cluster of non-necrotising nonfibrotic, well-circumscribed granulomas (GR) close to an elongated, lightly stained fibroblast plug that indicates involvement of an alveolar duct with organising pneumonia (OP). Haematoxylin and eosin staining.

were more frequent on CT in patients with CVID than in controls $(p=0.003)$ and represented the main CT pattern $(p=0.004)$. Halo signs were also more frequent, being found in only two $(3.5 \%)$ controls with sarcoidosis $(p=0.003)$. Whereas micronodules did not have a significantly different prevalence, they were more frequently the main CT pattern in sarcoid controls than in patients $(p=0.004)$. Moreover, the distribution of micronodules was not the same. In sarcoid controls, the micronodule distribution was perilymphatic in all cases while it was a random distribution for seven patients with ILD/CVID/GD $(\mathrm{p}<0.001)$. Bronchiectasis was involved more lobes (2.3 versus $1.4, \mathrm{p}=0.008)$ and more frequently in lower lobes (12 out of 13 versus six out of $13, \mathrm{p}=0.03$ ) in patients. Moreover, they were less likely to be traction bronchiectasis in ILD/CVID/GD patients than in sarcoid controls (two out of 13 versus seven out of $13, p=0.09$ ).

\section{Outcome and treatment}

Patients with ILD/CVID/GD and pulmonary sarcoidosis controls were followed for a median (range) time of 80.5 (7-201) and 68.5 (3-188) months, respectively. Treatment and evolution of patients and controls are reported in table $4.17(80 \%)$ patients were treated with immunoglobulin replacement. Patients were treated according to clinician judgement, at various stages of evolution and for various lengths of time, with corticosteroids $(n=15)$, immunosuppressive therapy $(n=3)$ and/or hydroxychloroquine $(\mathrm{n}=2)$ depending on symptoms, autoimmune manifestations and ILD severity and/or rate of worsening.

Regarding radiological findings, 17 patients with ILD/CVID/ GD had a follow-up CT after $53 \pm 45$ months. The main CT pattern improved in 10 patients, worsened in six patients and was stable in one patient. No patient had a complete resolution of pulmonary infiltration. A few intralobular reticulation or subpleural curvilinear lines, interpreted as discrete features of pulmonary fibrosis, were observed in five $(29 \%)$ patients. By comparison, in sarcoid controls, 36 patients had a follow-up chest CT after $42.5 \pm 29$ months. Four patients had a complete resolution of pulmonary infiltration. $18(50 \%)$ patients with

TABLE 3 Computed tomography (CT) findings at the time of diagnosis of interstitial lung disease (ILD) in common variable immunodeficiency disorder (CVID)-associated granulomatous disease (GD) and comparison with sarcoid controls

\begin{tabular}{|c|c|c|c|}
\hline & ILD/CVID/GD & Sarcoid controls & p-value \\
\hline Subjects & 20 & 57 & \\
\hline Halo sign & $6(30)$ & $2(3.5)$ & 0.003 \\
\hline Micronodules & $12(60)$ & $45(79)$ & 0.1 \\
\hline Distribution & & & $<0.001$ \\
\hline Lines & $13(65)$ & $33(58)$ & 0.58 \\
\hline Ground glass & $8(40)$ & $27(47)$ & 0.57 \\
\hline Airspace consolidation & $5(25)$ & $24(42)$ & 0.19 \\
\hline Bronchiectasis & $13(65)$ & $13(23)$ & $<0.001$ \\
\hline Bronchial wall thickening & $9(45)$ & $33(58)$ & 0.32 \\
\hline Mediastinal lymph nodes & $14(70)$ & $41(72)$ & 0.87 \\
\hline Micronodules & 5 & 20 & \\
\hline Ground glass & 1 & 15 & \\
\hline Lines & 4 & 3 & \\
\hline Consolidation & 0 & 9 & \\
\hline Miscellaneous ${ }^{\#}$ & 4 & 5 & \\
\hline
\end{tabular}

Data are presented as $n$ or $n(\%)$, unless otherwise stated. ${ }^{*}$ : lines/ground glass (three versus one), nodules/ground glass (one versus none) and bronchial wall thickening (none versus four). Bold indicates statistically significant $p$-values. 


\begin{tabular}{|c|c|c|c|}
\hline \multirow[t]{2}{*}{ TABLE 4} & \multicolumn{3}{|c|}{$\begin{array}{l}\text { Comparison between patients with interstitial } \\
\text { lung disease (ILD) in common variable } \\
\text { immunodeficiency disorder (CVID)-associated } \\
\text { granulomatous disease (GD) and sarcoid } \\
\text { controls: treatment and evolution. }\end{array}$} \\
\hline & & ILD/CVID/GD & Sarcoid controls \\
\hline \multicolumn{2}{|c|}{ Subjects $\mathrm{n}$} & 20 & 60 \\
\hline \multicolumn{2}{|c|}{ Follow-up months median (range) } & $80.5(7-201)$ & $69(12-188)$ \\
\hline \multicolumn{2}{|c|}{ Immunoglobulin replacement } & $17(85)$ & $0(0)$ \\
\hline \multicolumn{2}{|c|}{ Systemic treatment } & $16(80)$ & $56(93)$ \\
\hline \multicolumn{2}{|c|}{ Oral corticosteroids } & $15(75)$ & $46(77)$ \\
\hline \multicolumn{2}{|c|}{ Hydroxychloroquine } & $2(10)$ & $13(22)$ \\
\hline \multicolumn{2}{|c|}{ Immunosuppressive therapy } & $3(15)$ & $20(33)$ \\
\hline \multicolumn{2}{|c|}{ Fibrosis on follow-up CT scan" } & $5(29)$ & $18(50)$ \\
\hline \multicolumn{2}{|c|}{ Death $\star * *$} & $6(30)$ & $0(0)$ \\
\hline
\end{tabular}

sarcoidosis had new signs indicating pulmonary fibrosis progression $(\mathrm{p}=0.16)$.

At the end of follow-up, six (30\%) patients with ILD/CVID/ GD were dead but all of the pulmonary sarcoidosis controls were still alive. Three deaths were attributable to complications of CVID: 1) Streptococcus pneumoniae septic shock; 2) endstage obstructive pulmonary disease secondary to diffuse bronchiectasis; and 3) Epstein-Barr virus-associated peritoneal large B-cell lymphoma. Of the remaining three deaths, one was attributable to a myocardial infarction, one to meningoencephalitis of unknown cause and the other to acute respiratory insufficiency of unknown cause. Survival of patients was lower than that of sarcoid controls, with a probability of survival of $79 \%$ versus $100 \%$ at 10 yrs $(p=0.004)$ (fig. 4$)$.

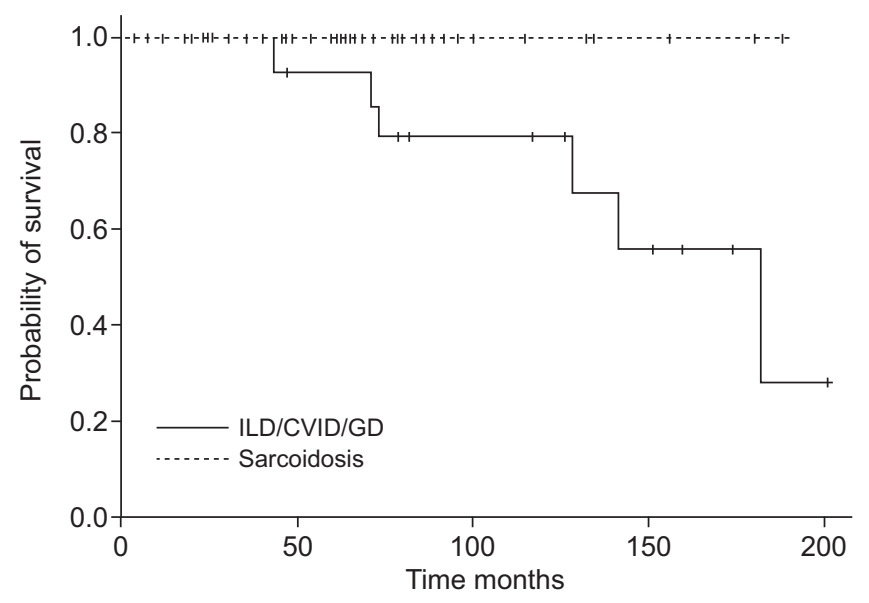

FIGURE 4. Kaplan-Meier overall survival for interstitial lung disease (ILD) in common variable immunodeficiency disorder (CVID)-associated granulomatous disease (GD) patients and sarcoidosis controls.

\section{DISCUSSION}

The present study is the first case-control series comparing patients with ILD/CVID/GD and pulmonary sarcoidosis. The main results were: 1) lung manifestations were definitely different from patients with pulmonary sarcoidosis in almost all cases, taking into account clinical history, physical examination, CT imaging and BAL cell count; 2) the longstanding evolution of ILD was marked by the absence of any trend toward spontaneous recovery or significant fibrosis; and 3) poor general survival and morbidity were linked mainly to CVID, through infection, bronchiectasis and lymphoproliferative disorders. Moreover, the lung interstitial pathological lesions most often consisted of granulomatous lesions but, in some patients, did contain some degree of CVID-linked lymphoid infiltration.

The occurrence of noncaseating granulomatous lesions in patients with CVID has previously been well described [7-13]; whether it should be considered as sarcoidosis or "sarcoid-like" process is still debated $[5,7,9,10]$. In the literature, as in our study, key phenotypic differences between CVID/GD and sarcoidosis have been pointed out. Some of them stem from CVID, like a high frequency of recurrent infections responsible for a particularly high frequency of bronchiectasis and autoimmune manifestations.

A painstaking investigation of clinical, CT and BAL manifestations, we report here evidence of some more obvious differences between CVID/GD and pulmonary sarcoidosis. Velcro crackles, uncommon in pulmonary sarcoidosis, were noted in half of our CVID/GD patients. BAL was available in 14 of our patients; even though lymphocytosis was found in most cases, as in sarcoidosis, a decreased CD4/CD8 T-cell ratio, which is very rare in sarcoidosis [16], was found in $50 \%$ of cases, while only one patient had a ratio $>3.5$, a manifestation commonly found in sarcoidosis and considered as highly suggestive of this diagnosis [17].

Some studies [11, 12, 18] had suggested that one important difference between CVID/GD and pulmonary sarcoidosis was the CT scan features and, particularly, the aspect and distribution of nodules and micronodules. Our series clearly confirmed their findings. In our series, only $10 \%$ of cases mimicked sarcoidosis, by typical bilateral hilar lymphadenopathy and mainly lymphatic-distributed diffuse lung micronodules [19]. Most frequently, CT showed peripheral nodules with air bronchograms and smooth margins surrounded by ground-glass opacities (halo sign), as well as random distribution of micronodules; all of which would look very atypical in sarcoidosis. The other manifestations considered atypical for sarcoidosis were the frequency and type of bronchiectasis and the scarcity of fibrosis.

The CT halo sign was found in as much as 35\% of our patients, which is noteworthy. In sarcoidosis, the halo sign is rarely found [20, 21]. HARADA et al. [20] reported the only case showing a pathological/radiological in this setting. The solid area of the lesion contained epithelioid cell granulomas. The ground-glass area also corresponded to granulomas associated with mononuclear cells localised in the peripheral airways and alveolar spaces. In our series, a pathological analysis was possible on three out of six patients with nodules surrounded 
by ground-glass opacity. It unveiled isolated granulomatous lesions in two cases and a solid area of granulomas surrounded by a peripheral area showing organising pneumonia in one.

Ultimately, the pathological findings were not revealed to be strictly similar. Even though typical granulomatous lesions, without different features and with sarcoid granulomas, could be evidenced in six out of seven lung biopsies, no less than three cases also contained other lesions, either associated or alone. These associated or isolated lesions consisted of organising pneumonia or follicular bronchiolitis, two lesions well known to be associated with CVID [7, 18, 22, 23] and recently regrouped under the term GLILD (granulomatouslymphocytic ILD) [7, 24]. Moreover, the risk of mucosaassociated lymphoid tissue lymphoma could be increased in CVID and appeared as a consolidation on CT scans, a presentation that has been described in lymphoma in other contexts [25]. Thus, if granulomatous lesions appear a plausible pathological explanation for nodules or consolidations, other pathological diagnostic hypothesis cannot be definitively ruled out even when GD is confirmed in extrapulmonary samples.

In the literature, CVID/GD is considered to have a poor prognosis [7, 10]. In their analysis of the files of 69 patients with CVID, BATES et al. [7] found a reduction in median survival by $>50 \%$, to about 14 yrs in case of granulomatouslymphocytic lung disease compared with a median survival of nearly 30 yrs for patients with CVID alone. In this series, the mechanisms of fatal evolution were not specified. In the series of MECHANIC et al. [10], the outcome was also severe with a comparable mortality. Four out of 17 patients died of end-stage restrictive pulmonary disease, the mechanism being imprecisely specified, while one patient had severe hepatic granulomatosis due to ascites, hepatic encephalopathy and portal hypertension [10]. However, ARDENIZ and CUNNINGHAMRUNDLES [5] found a similar mortality between CVID patients with and without GD. Among the 10 deaths, six were related to granulomatous lung disease, complicated by pneumonia in three cases [10]. In our series, the high mortality rate of $30 \%$ with a survival of $79 \%$ at $10 \mathrm{yrs}$ was in line with this. Interestingly, this high mortality rate was not due to the progression of ILD nor to the GD per se, perhaps because most of our patients benefited from immunoglobulin substitution and corticosteroids or immunosuppressive treatments.

There are several limitations to our study. First, as it was retrospective, some data were missing. For example, a pathological analysis of the lung was not made in all our patients and the real proportion of cases with granulomas and/or other lymphoid lesions remains unknown. In particular, we could not definitely give the signification of CT elementary lesions. The impact of treatment on GD and ILD could not be evaluated. However, conversely to Vultagio et al. [13], we did not observe any increased risk of infection after immunosuppressive regimens.

In summary, ILD/CVID/GD is a clinical entity distinct from sarcoidosis. Autoimmune disorders, crackles, micronodules with a random distribution and nodules with halo signs were not or only unusually found in the sarcoid controls. So, in patients with suspected sarcoidosis, the presence of these clinical or radiological findings should raise a strong suspicion of underlying CVID.

ILD was not a critical determinant of prognosis of these patients. Conversely, the poor prognosis was linked to classical complications of CVID (lymphoma, death due to infection or obstructive lung disease secondary to bronchiectasis), which could be more frequent in the case of an associated GD. In addition, because of frequently associated lymphoid lesions, lung pathological analysis should be discussed in some CT presentations. Finally, continuing reporting GD associated CVID seems important to improve our knowledge of this disease, and especially the mechanisms involved in the granulomatous formation.

\section{STATEMENT OF INTEREST}

None declared.

\section{ACKNOWLEDGEMENTS}

The authors affiliations are as follows. D. Bouvry: Assistance Publique - Hôpitaux de Paris (AP-HP), Service de Pneumologie, and Université Paris 13, PRES Sorbonne-Paris-Cité, EA 2363, Hôpital Universitaire Avicenne, Bobigny, France. L. Mouthon: Université Paris Descartes, Faculté de Médecine, Pôle de Médecine interne, hôpital Cochin, and Institut Cochin, INSERM U1016, CNRS UMR 8104, Paris, France. P-Y. Brillet: AP-HP, Service de Pneumologie, Hôpital Universitaire Avicenne, and AP-HP, Service de Radiologie, Hôpital Universitaire Avicenne. M. Kambouchner: AP-HP, Service d'Anatomie Pathologie, Hôpital Universitaire Avicenne. J-P. Ducroix: Service de Médecine Interne, Hôpital Universitaire Nord d'Amiens, Amiens, France. V. Cottin: Service de Pneumologie, Hôpital Universitaire Louis Pradel, Hospices Civils de Lyon, Lyon, France. J. Haroche: AP-HP, Service de Médecine Interne, Hôpital Universitaire Pité-Salpètrière, Paris. J-F. Viallard: Service de Médecine Interne et des Maladies Infectieuses, Hôpital Universitaire Haut-Lévêque, Bordeaux, France. R. Lazor: Service de Pneumologie, Hôpital Universitaire Vaudois, Lausanne, Switzerland. F. Lebargy: Service de Pneumologie, Hôpital Universitaire Maison Blanche, Reims, France. A. Tazi: AP-HP, Service de Pneumologie, Hôpital Universitaire Saint Louis, Paris. B. Wallaert: Service de Pneumologie et Immuno-Allergologie, Hôpital Universitaire Calmette, Lille, France. A. Smail: Service de Médecine Interne, Hôpital Universitaire Nord d'Amiens. J-L. Pellegrin: Service de Médecine Interne et des Maladies Infectieuses, Hôpital Universitaire HautLévêque. H. Nunes: Assistance Publique - Hôpitaux de Paris (AP-HP), Service de Pneumologie, and Université Paris 13, PRES SorbonneParis-Cité, EA 2363, Hôpital Universitaire Avicenne. Z. Amoura: AP-HP, Service de Médecine Interne, Hôpital Universitaire PitéSalpètrière. J-F. Cordier: Service de Pneumologie, Hôpital Universitaire Louis Pradel, Hospices Civils de Lyon. D. Valeyre: AP-HP, Service de Pneumologie, and Université Paris 13, PRES SorbonneParis-Cité, EA 2363, Hôpital Universitaire Avicenne. J-M. Naccache: AP-HP, Service de Pneumologie, and Université Paris 13, PRES Sorbonne-Paris-Cité, EA 2363, Hôpital Universitaire Avicenne.

We thank V. Levy (Unité de Recherche Clinique, Hôpital Avicenne, Bobigny, France) for his statistical assistance.

\section{REFERENCES}

1 Conley ME, Notarangelo LD, Etzioni A. Diagnostic criteria for primary immunodeficiencies. Clin Immunol 1999; 93: 190-197.

2 Chapel H, Lucas M, Lee M, et al. Common variable immunodeficiency disorders: division into distinct clinical phenotypes. Blood 2008; 112: 277-286.

3 Cunningham-Rundles C, Bodian C. Common variable immunodeficiency: clinical and immunological features of 248 patients. Clin Immunol 1999; 92: 34-48. 
4 Park MA, Li JT, Hagan JB, et al. Common variable immunodeficiency: a new look at an old disease. Lancet 2008; 372: 489-502.

5 Ardeniz O, Cunningham-Rundles C. Granulomatous disease in common variable immunodeficiency. Clin Immunol 2009; 133: 198-207.

6 Oksenhendler E, Gerard L, Fieschi C, et al. Infections in 252 patients with common variable immunodeficiency. Clin Infect Dis 2008; 46: 1547-1554.

7 Bates CA, Ellison MC, Lynch DA, et al. Granulomatous-lymphocytic lung disease shortens survival in common variable immunodeficiency. J Allergy Clin Immunol 2004; 114: 415-421.

8 Arnold DF, Wiggins J, Cunningham-Rundles C, et al. Granulomatous disease: distinguishing primary antibody disease from sarcoidosis. Clin Immunol 2008; 128: 18-22.

9 Fasano MB, Sullivan KE, Sarpong SB, et al. Sarcoidosis and common variable immunodeficiency. Report of 8 cases and review of the literature. Medicine (Baltimore) 1996; 75: 251-261.

10 Mechanic LJ, Dikman S, Cunningham-Rundles C. Granulomatous disease in common variable immunodeficiency. Ann Intern Med 1997; 127: 613-617.

11 Park JE, Beal I, Dilworth JP, et al. The HRCT appearances of granulomatous pulmonary disease in common variable immune deficiency. Eur J Radiol 2005; 54: 359-364.

12 Torigian DA, LaRosa DF, Levinson AI, et al. Granulomatouslymphocytic interstitial lung disease associated with common variable immunodeficiency: CT findings. J Thorac Imaging 2008; 23: 162-169.

13 Vultaggio A, Matucci A, Parronchi P, et al. Association between "sarcoidosis-like" disease and common variable immunodeficiency (CVI): a new CVI variant showing an activation of the immune system. Sarcoidosis Vasc Diffuse Lung Dis 2007; 24: 127-133.

14 Statement on sarcoidosis. Joint Statement of the American Thoracic Society (ATS), the European Respiratory Society (ERS) and the World Association of Sarcoidosis and Other Granulomatous Disorders (WASOG) adopted by the ATS Board of Directors and by the ERS Executive Committee, February 1999. Am J Respir Crit Care Med 1999; 160: 736-755.

15 Hansell DM, Bankier AA, MacMahon H, et al. Fleischner Society: glossary of terms for thoracic imaging. Radiology 2008; 246: 697-722.

16 Agostini C, Trentin L, Zambello R, et al. CD8 alveolitis in sarcoidosis: incidence, phenotypic characteristics, and clinical features. Am J Med 1993; 95: 466-472.

17 Welker L, Jorres RA, Costabel U, et al. Predictive value of BAL cell differentials in the diagnosis of interstitial lung diseases. Eur Respir J 2004; 24: 1000-1006.

18 Tanaka N, Kim JS, Bates CA, et al. Lung diseases in patients with common variable immunodeficiency: chest radiographic, and computed tomographic findings. J Comput Assist Tomogr 2006; 30: 828-838.

19 Nunes H, Brillet PY, Valeyre D, et al. Imaging in sarcoidosis. Semin Respir Crit Care Med 2007; 28: 102-120.

20 Harada T, Nabeshima K, Matsumoto T, et al. Histological findings of the computed tomography halo in pulmonary sarcoidosis. Eur Respir J 2009; 34: 281-283.

21 Marten K, Rummeny EJ, Engelke C. The CT halo: a new sign in active pulmonary sarcoidosis. Br J Radiol 2004; 77: 1042-1045.

22 Busse PJ, Farzan S, Cunningham-Rundles C. Pulmonary complications of common variable immunodeficiency. Ann Allergy Asthma Immunol 2007; 98: 1-8.

23 Wislez M, Sibony M, Naccache JM, et al. Organizing pneumonia related to common variable immunodeficiency. case report and literature review. Respiration 2000; 67: 467-470.

24 Park JH, Levinson AI. Granulomatous-lymphocytic interstitial lung disease (GLILD) in common variable immunodeficiency (CVID). Clin Immunol 2010; 134: 97-103.

25 Wislez M, Cadranel J, Antoine M, et al. Lymphoma of pulmonary mucosa-associated lymphoid tissue: CT scan findings and pathological correlations. Eur Respir J 1999; 14: 423-429. 